\title{
Microstructural and Fractographic Analysis of an Oil Pipeline Failed in Service
}

\author{
A. C. Vidal*, A. R. Martins* and F. A. Darwish** \\ * Institute of Technology, Catholic University of Rio de Janeiro, Rua Marquês de São Vicente, 225, \\ Gávea, 22453-900, Rio de Janeiro, Brasil \\ ** Department of Materials Science and Metallurgy, Catholic University of Rio de Janeiro, Rua \\ Marquês de São Vicente, 225, 22453-900, Rio de Janeiro, Brasil
}

A part of an oil pipeline, seated on the ocean floor, floated partially and was accidentally impacted by a passing motorboat, which ended up sinking as a result of the accident. In an effort to restore the pipeline back to its original position in the seabed, pressurized water was injected. As a result of this operation, water flushed out from a leaking section of the pipe, close to the impacted area.

The part of the pipe received for analysis measured about $4 \mathrm{~m}$ in length and contained a helicoidal welded seam with a large crack propagating circumferentially. It was observed that crack propagation had occurred through the metal base as well as along the heat-affected zone accompanied by intense plastic deformation in the impacted region.

Room temperature Charpy impact tests, were carried out on specimens machined from the metal base, heat-affected zone and fusion zone. The results obtained indicated impact energy levels of $7,41,23,6$ and $52,4 \mathrm{~J}$, respectively.

The base metal, an API 5L carbon steel, showed a microstructure consisting of ferrite grains together with strongly aligned pearlite colonies (Fig. 1). The heat-affected zone, close to the fusion line, presented a large amount of Widmannstäten ferrite (Fig. 2), while acicular ferrite was the predominant constituent in the weld metal (Fig. 3). These microstructural observations appear to be consistent with the impact results as well as the fractographic analysis carried out on specimens taken from the fractured part of the pipe.

The low impact resistance of the base metal is confirmed by the predominance of cleavage as the operating fracture mechanism (Fig. 4), consistent with the presence of aligned pearlite colonies. The absence of this aligned constituent in the heat-affected zone seems to be in good agreement with its higher impact resistance in comparison with that of the base metal.

Compared to the base metal, the fractographic results are indicative of the tougher behavior of the heat-affected zone, where one can notice, in Fig. 5, the presence of mixed fracture modes (cleavage facets together with dimples), indicating higher plastic deformation levels during the fracture process. The acicular ferrite phase, predominant in the fusion zone, presents good impact toughness properties, consistent with the high impact energy level exhibited by the Charpy specimens. This is confirmed by the predominance of ductile failure mechanism as shown by the presence of dimples (Fig. 6) on the fracture surface of specimens pertaining to the weld metal.

Finally, it is concluded that the impact results are compatible with the visual observations where the primary crack did in fact propagate through the base metal and along the heat-affected zone and never through the weld metal. 


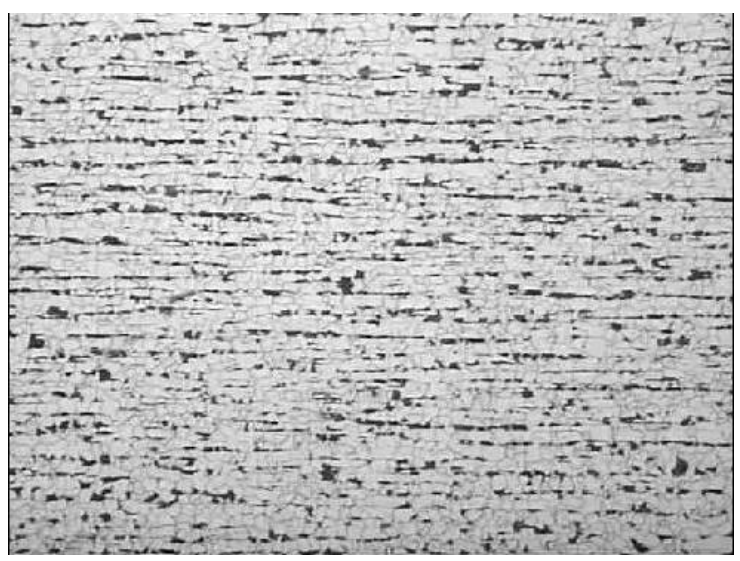

FIG. 1. Pipeline base metal. Presence of ferrite and aligned pearlite colonies. $25 \mathrm{X}$.

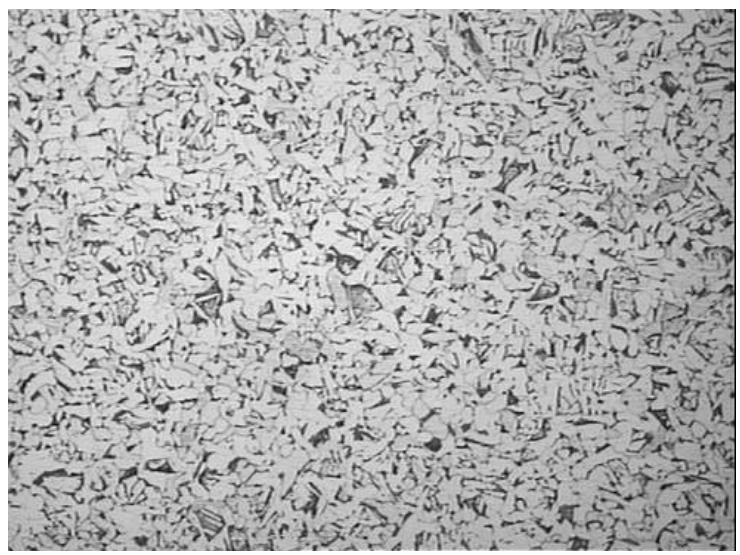

FIG. 3. Fusion zone of the welded joint. FIG. 4. Cleavage features predominating in the Predominance of acicular ferrite. 100X.

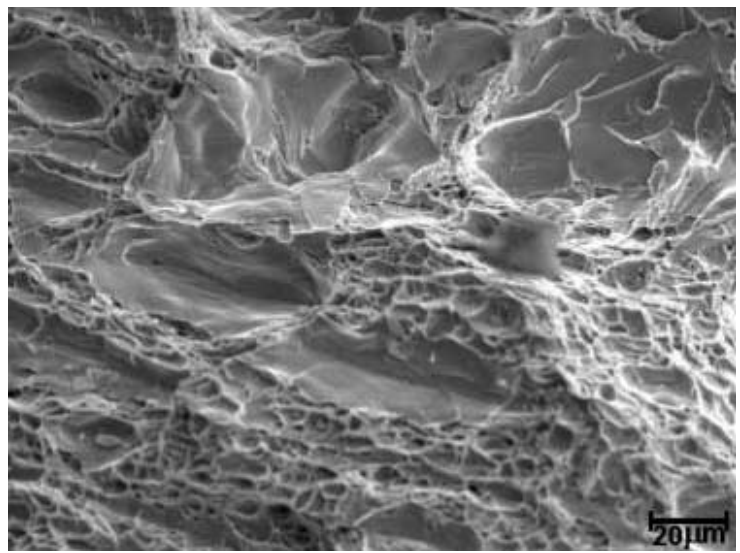

FIG. 5. Heat-affected zone fracture. Mixed fracture mode. Dimples and cleavage facets.

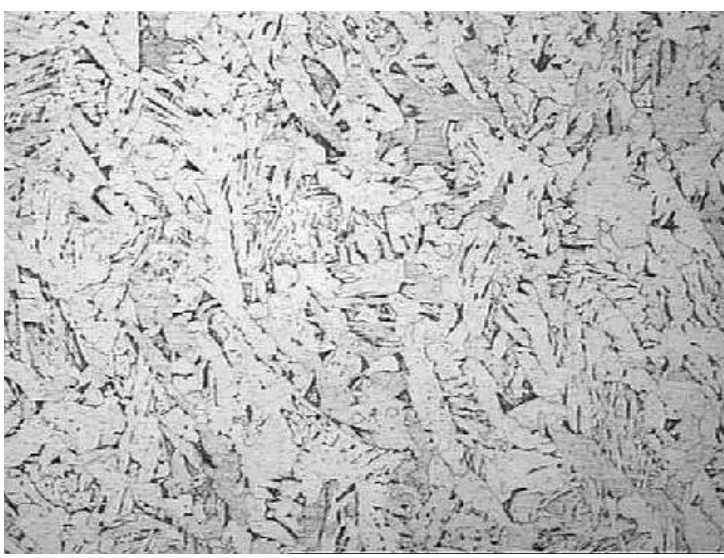

FIG. 2. Heat-affected zone of the welded joint. Widmannstäten ferrite. 100X.

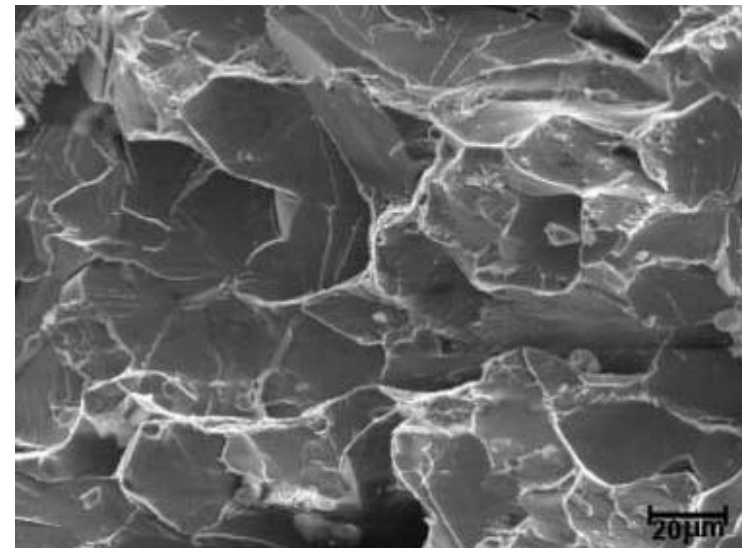
base metal fracture surface.

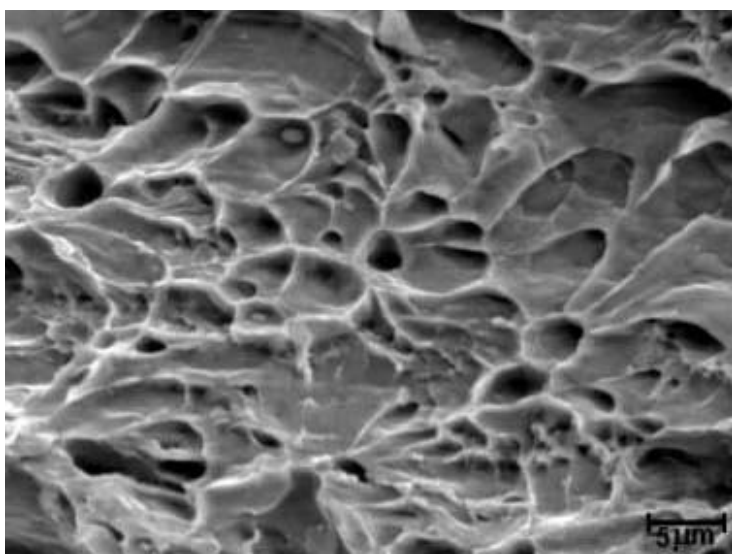

FIG. 6. Fracture surface of the fusion zone. Presence of dimples. 